\title{
Sensitization to apomorphine in pigeons is due to conditioning, subject to generalization but resistant to extinction
}

\author{
A.M. Godoy and J.D. Delius \\ Allgemeine Psychologie, Universität Konstanz, Konstanz, Germany \\ Correspondence to J.D. Delius, Allgemeine Psychologie, Universität Konstanz, D-78457 Konstanz, Germany. E-mail: \\ juan.delius@uni-konstanz.de
}

\begin{abstract}
Apomorphine (Apo) administration induces a persistent bout of pecking in pigeons and other birds. Repeated injections of Apo in pigeons lead to sensitization, i.e. the pecking response to a particular dose increases up to a dose-dependent asymptotic level. It is also known that Apo-induced pecking can be classically conditioned to the cage environment where the animals experience the effect of the drug. Here we address the question of whether, and to what extent, the sensitization effect arises as a consequence of a conditioning or of a pharmacological process. An extinction experiment demonstrated that an extinction procedure supposed to be effective in inhibiting the conditioned pecking response was not effective in suppressing the sensitization to Apo, thus casting provisional doubt on the conditioning hypothesis. However, a conditioning experiment demonstrated that the sensitization effect undoubtedly involved an important component of conditioning to an experimental cage environment, but also suggested that there was an additional component possibly not due to learning. A generalization experiment, however, showed that this second component was very probably due to a stimulus generalization effect deriving from conditioning to the home cage, suggesting that learning can account for most, if not all, of the increase in Apo-induced pecking and that an exclusively pharmacological sensitization process plays, at best, a minor role. The apparent contrast between the results of the first experiment, indicating that the sensitization is not affected by inhibitory conditioning, and the results of the last two experiments, suggesting that the sensitization is due to excitatory conditioning, can be resolved by assuming that Apo induces a drug-state-dependent conditioning. These results are related to findings and arguments concerning the sensitization to psychostimulant drugs in mammals. (C) 1999 Lippincott Williams \& Wilkins.
\end{abstract}

Keywords: pigeon, apomorphine, dopamine, pecking, sensitization, conditioning, extinction, generalization

\section{INTRODUCTION}

Apomorphine (Apo) is known to be a potent, direct, mainly post-synaptically acting, agonist at dopamine receptors (Kruk and Pycock, 1991). Among certain other responses, Apo administration elicits oral stereotypies in many different species such as snails, toads, fish, tortoises, mice, rats and humans (cf. Fekete et al., 1970; Andersen et al., 1975; Ljungberg and Ungerstedt, 1977; Szechtman et al., 1987; Glagow and Ewert, 1997). In pigeons, chickens and other birds an optimal intramuscular dose of Apo yields an impressive bout of repetitive pecking beginning within a few minutes, lasting for about $1 \mathrm{~h}$ and involving several thousand pecks (Brunelli et al., 1975; Machlis, 1980). The administration of amphetamine, but apparently not that of cocaine, elicits a much weaker pecking bout (Cheng et al., 1975). The response to Apo exhibits a dose-dependent increase, from about $0.1 \mathrm{mg} / \mathrm{kg}$ up to about $2 \mathrm{mg} / \mathrm{kg}$ where a ceiling is reached. At higher doses coordination impairments begin to interfere with the pecking response. Except with the lowest doses, which also yield some preening, the response to Apo is largely restricted to pecking. Control saline (Sal) injections do not yield more than a dozen occasional pecks (Basten-Kreft, 1977). Pecking is a quite specific response, only elicited by a restricted range of stimulus conditions (Delius, 1985; Siemann and Delius, 1992a). Although the Apo pecks are very similar in pattern to foraging pecks (Siemann and Delius, 1992b), and are sometimes directed at grains, they do not usually lead to grain ingestion, Apo having a strong anorexic effect (Deviche, 1984). Even though Apo also induces pecking in total darkness (Leydel and Delius, unpublished experiment 1999), under usual conditions the pecks are directed at small contrasting visual features. These can be dark dots on the light walls of experimental cages, cross-welds of their home cages, dust-specks on plain surfaces or 
even the claws of the pigeons' own feet (Brunelli et al., 1975; Basten-Kreft, 1977). Apo administration reliably gives rise to sensitization, in the sense that, when pigeons are repeatedly injected on a daily schedule with a given dose of Apo, their pecking response increases cumulatively with each injection, up to a dose-dependent asymptote. This sensitization occurs over a range of doses, from $0.2 \mathrm{mg} / \mathrm{kg}$ up to at least $1.4 \mathrm{mg} / \mathrm{kg}$ Apo, within some three-six daily injections, and entails an increase up to about six- to eight-fold in peck response (Basten-Kreft, 1977; Wynne and Delius, 1995; see also below).

In mice, rats and other mammals, the most salient response to Apo administration is an increase in locomotor activity. When the animals have previously been subjected to a unilateral 6-hydroxy-dopamine (6-OHDA) lesion of the substantia nigra, this response becomes a turning response. Repeated Apo injections yield sensitization with respect to these responses in rats (Carey, 1986; Mattingly and Gotsick, 1989; Mattingly et al., 1997). However, injections of amphetamine and cocaine elicit analogous responses and a similar sensitization (Anagnostaras and Robinson, 1996; Burechailo and MartinIverson, 1996; Kuczenski et al., 1997; White et al., 1998). The detailed mechanism by which the sensitization to all these drugs comes about is a much disputed issue. Some authors believe that pharmacological mechanisms (drug accumulation; increase in dopamine receptors) are responsible or at least partly implicated; other authors consider that only, or mainly, conditioning processes are involved, but without agreeing about precisely which ones (Kalant, 1989; Pert et al., 1990; Stewart and Vezina, 1991; Kuribara and Uchihashi, 1993; Stewart and Badiani, 1993; Anagnostaras and Robinson, 1996; Bedingfield et al., 1996; Burechailo and Martin-Iverson, 1996; Crombagh et al., 1996; Lienau and Kuschinsky, 1997; Mattingly et al., 1997). It is possible that the sensitization mechanisms to these drugs are not exactly the same as amphetamine and cocaine, and unlike Apo, are rather unspecific, indirect and presynaptically acting agonists of dopamine. The fact that cocaine and amphetamine can produce drug dependence but apomorphine does not, suggests that they are not all fully equivalent in their behavioural effects (Julien, 1995). It may even be that the locomotor and turning responses in rodents, even if both are not particularly specific responses, are not identical with respect to the sensitization mechanisms (Schwarting and Huston, 1996).

The Apo-elicited pecking of pigeons can, in any case, be conditioned to visually distinctive cages. Pigeons repeatedly treated with Apo in one such cage and with Sal in another pecked significantly more in the first cage than in the second when subsequently tested under exclusive Sal treatment. That is, an Apo injection acts as an unconditioned stimulus (US) which elicits a delayed unconditioned response (UR pecking). When the US is repeatedly paired with a distinctive cage (conditioned stimulus, CS), this treatment causes that CS to elicit a conditioned response (CR pecking; Lindenblatt and Delius, 1987; cf. Mattingly and Gotsick, 1989: rats). In agreement with this Pavlovian schema, the pigeons also show a progressive shortening of pecking response latencies with successive CS-US pairings, as the CR pecking begins to precede the UR pecking (Wynne and Delius, 1995; cf. Carey, 1986: rats). Because Lindenblatt and Wynne (1987) used a balanced differentiation paradigm it is reasonably certain that the pecking CRs were not artefactually due to non-associative processes such as behavioural sensitization or habituation to the test procedures and environments. Burg et al. (1989), using a discriminative place conditioning procedure, additionally showed that Apo administration has a rewarding effect in pigeons (cf. Bell et al., 1997: rats).

The main purpose of the present study was to reassess the validity of an increment-through-associative-conditioning account for the sensitization to Apo in pigeons proposed by Burg et al. (1989). These authors found that sensitization to repeated Apo injections only became apparent in the same distinctive cage in which the pigeons had previously experienced the Apo treatment, but that it was absent in another distinctive cage in which they had previously experienced Sal injections (cf. Stewart and Vezina, 1991: rats). The response increments thus seemed to emerge because, due to repeated environment-drug (CS-US) pairings, the relevant cage environment began to elicit an increasing CR pecking that summated to the UR pecking directly elicited by Apo, resulting in a unified CR plus UR pecking. Alternative non-associative explanations, i.e. in terms of novelty/familiarity with the testing environments and procedures, was again largely precluded by the use of a differentiation-type procedure. However, because of design limitations the magnitude of the sensitization effect studied by Burg et al. (1989) was small, involving only a fraction of the complete effect. The CRs obtained by Lindenblatt and Delius (1987) were also too small to account for the total sensitization increases, and Wynne and Delius (1995) found that Apo sensitization was resistant to latent inhibition, a CS-no-US pre-treatment, otherwise known to reduce subsequent Pavlovian conditioning (Domjan, 
1993). These findings made it necessary to reexamine the conditioning hypothesis with three experiments.

If the sensitization that arises with repeated administrations of Apo is mainly due to a classical conditioning process, it is expected to be affected by the same kinds of treatments that are known to regularly affect standard Pavlovian conditioning. Among the most reliable characteristics of conditioned responses is that they are susceptible to a form of inhibitory conditioning, namely extinction. That is, any $\mathrm{CR}$ to a CS that has arisen through repeated CSUS pairings is expected to progressively weaken after a subsequent series of CS-no-US presentations (Domjan, 1993). An extinction experiment explored whether such a treatment would similarly suppress sensitization to Apo previously acquired in a distinctive cage environment.

The notion that conditioning plays an important role in the development of sensitization to Apo in pigeons primarily relies, as already mentioned, on the result of an experiment by Burg et al. (1989). It concerned the sensitization which occurred between a second and third daily Apo injection and, accordingly, relied on a small response difference between experimental and control groups. Normally, some four consecutive injections are actually required to guarantee a fully asymptotic response to the relevant $0.5 \mathrm{mg} / \mathrm{kg}$ Apo dose (Acerbo et al., 1997; see also below). The present conditioning experiment was meant to re-examine the validity of the conditioning hypothesis using a different procedure, which ensured a complete course of sensitization and which included a more comprehensive set of control treatments.

In the event, the latter experiment suggested that sensitization to Apo is partially due to conditioning. However, it left open the question of whether part of the effect obtained was due to a pharmacological process unrelated to learning or whether it could still be due to a process of conditioning plus generalization. This question arose because part of the CR that would have developed to the home cage during training might have transferred to the experimental cage during testing, due to the physical similarity between both cages. A generalization experiment was designed to assess the role of such stimulus generalization. For that purpose we compared the sensitization transfer that pigeons would show when tested either in the same experimental cage as before, or in an experimental cage very different from the home cage.

\section{METHODS}

\section{Subjects}

Adult pigeons (Columba livia), bred from local homing stock and weighing between 450 and $550 \mathrm{~g}$ were used. A week before the experiments began they were moved from an outside aviary to individual $40 \times 40 \times 45 \mathrm{~cm}$ stainless steel grid cages. These home cages were located in a well-ventilated and brightly lit ( $12 \mathrm{~h}$ on-12h off) room. Animal maintenance and treatments conformed to the standards and rules laid down by the German animal protection laws and regulations.

\section{Procedure}

Each experiment consisted of a series of daily treatments. For each of these treatments the pigeons were injected with either an Apo solution or physiological saline (Sal) into the pectoral muscles, or left untreated. The treatment schedules and drug doses used are specified below. The pigeons were immediately placed either back into their home (Ho) cages or alternatively into experimental cages to be described below. When placed into the experimental cages, but not when placed into the Ho cages, the pigeons were videotaped for $20 \mathrm{~min}$ before they were returned to their Ho cages. The videotapes were later reviewed and the number of pecks recorded. Because these response scores were mostly not normally distributed they were analysed with either the withinor the between-subjects version of the two-sample non-parametric permutation test for differences between means (Siegel and Castellan, 1988). These tests yield probability estimates without any intermediate statistics. The significance of combined group differences were assessed with the procedure recommended by Winer (1971, p. 49).

\section{Extinction}

Twelve pigeons underwent a sensitization training phase. They were injected with $1.0 \mathrm{mg} / \mathrm{kg}$ Apo and placed into a distinctive (Di) cage (paired CS-US condition) for six daily standard sessions. The Di cage was a modified standard grid cage with the inner surfaces of the back and side walls lined with white painted panels sprinkled with dark green dots $\left(0.8 \mathrm{~mm}\right.$ in diameter, about 10 per $\left.100 \mathrm{~cm}^{2}\right)$. After training the birds were randomly divided into two groups of six pigeons each. An extinction phase followed, consisting of six sessions during which all the pigeons were injected with Sal. The animals belonging to the extinction group (Ext) were placed in the Di cage. The repeated exposure to this cage 
without the Apo US was expected to weaken the pecking CR to the relevant CS. Birds in the control group (Cnt) were simply returned to their Ho cages and thus not subjected to the extinction procedure. A testing phase consisting of three daily sessions followed. For every test session all of the animals were injected with Apo and placed into the Di cage.

\section{Conditioning}

Twenty-five adult pigeons were randomly allocated to five groups $(n=5)$. There were seven daily sessions of training and three daily sessions of testing. For the training sessions each pigeon was injected with either Apo $(0.5 \mathrm{mg} / \mathrm{kg})$ or an equal volume of Sal and placed into either their Ho cage or the Di cage described earlier. Hoping for a better differentiation between conditioned vs unconditioned effects, a smaller dose of Apo than previously was used. Pigeons in the ApoDi group were injected with Apo and placed into the Di cage. In conditioning terms this group consistently experienced paired CS-US presentations considered to be essential for the development of a CR. Birds in the Di-Apo group were first placed into the Di cage for the standard 20-min period, without any prior injection, and then returned to their Ho cages. Randomly between 1 and $3 \mathrm{~h}$ later these animals were injected with Apo and returned again to their Ho cages. Thus, this group experienced systematically unpaired CS-US presentations, the CS preceding the US by an interval considered too long and too varied to normally support excitatory conditioning (Domjan, 1993). Pigeons in the Sal-Di group were injected with Sal and immediately placed into the Di cage so that, in conditioning terms, they only received CS exposures, in order to control for any non-associative cage familiarization. Birds in the Apo-Ho group were injected with Apo and placed back into their Ho cages. The pigeons of this group were thus only subjected to the US in their Ho cages and were not expected to develop a CR in response to the Di cage. Note that, according to a pharmacological hypothesis, all of the groups exposed to the drug regardless of whether paired with the experimental cage or not (Apo-Di, Di-Apo and Apo-Ho groups) should develop an approximately equivalent sensitization to Apo during the training phase. According to the conditioning hypothesis, however, these paired and unpaired contingencies of Apo and Di cage should, of course, yield differing results. Birds in the Sal-Ho group were injected with Sal and stayed in their Ho cage, and were thus kept naive to both the CS and the US. They could also serve as controls for a possible CS familiarization effect.
The test phase began on the day following the last training session. For the three sessions of this phase all pigeons, irrespective of group, were injected once daily with Apo and placed into Di cages. Note that the complete labelling of the six groups listed above should therefore really be Xxx-Yy/Apo-Di, with the oblique stroke separating the training from the testing conditions. This remark will be helpful when we deal with the next experiment. Note also that although there were three testing sessions, only the first one was adequately informative about the effects that the training treatments had had on the response to Apo in the Di cage. The sensitization up to some asymptote that was expected to develop during the test sessions in all groups except the already sensitized Apo-Di group would tend to obscure the initial inter-group differences. The purpose of the second and third test sessions under the Apo-Di was only to ascertain that such sensitization did in fact take place. The reader should, incidentally, be wary of any comparisons between session responses or sensitization courses obtained during the test phase, and responses or courses recorded for the Apo-Di group at the beginning of training. Besides the possibility that the scores being compared would be likely to be influenced by seasonal trends, more marked in pigeons than in rodents, they would also refer to pigeons that had unequal experience with the injection procedure.

\section{Generalization}

Twenty new adult pigeons were randomly divided into two groups $(n=10)$. The experiment consisted of nine daily training sessions and three daily testing sessions. During the training phase one group of pigeons was injected with $0.5 \mathrm{mg} / \mathrm{kg}$ Apo and returned to the Ho cage (training equivalent to that of group Apo-Ho in the former experiment). The other group was injected with Sal and also returned to the Ho cage (training equivalent to that of group Sal-Ho in the previous experiment). As in the earlier experiments the behaviour in the Ho cages was not recorded. The first group was, as previously explained, expected to develop sensitization to Apo in the Ho cages, the second group served as a control. For the subsequent test phase all pigeons were injected with $0.5 \mathrm{mg} / \mathrm{kg}$ Apo and placed into one of two different test cages. Half of the Apo-Ho and half of the Sal-Ho trained pigeons were tested in the same Di cage used in the conditioning experiment; these groups were thus equivalent to the Apo-Ho/Apo-Di and $\mathrm{Sal}-\mathrm{Ho} / \mathrm{Apo}-\mathrm{Di}$ groups of that experiment. The other half of the pigeons were tested in a new 
aberrant $(\mathrm{Ab})$ cage and thus constituted novel Apo$\mathrm{Ho} / \mathrm{Apo}-\mathrm{Ab}$ and $\mathrm{Sal}-\mathrm{Ho} / \mathrm{Apo}-\mathrm{Ab}$ groups. To abbreviate further references to the above groups they are henceforth called Apo/Di, Sal/Di, Apo/Ab and Sal/ $\mathrm{Ab}$, respectively. The $\mathrm{Ab}$ cage was a cylindrical, metallic sheet enclosure $(45 \mathrm{~cm}$ diameter) with a wooden back wall, a wooden floor and a wire-netting front wall. The inside was painted white and sprinkled with dark green dots, as in the Di cages. Note that this latter similarity between the $\mathrm{Di}$ and $\mathrm{Ab}$ cages cannot have influenced the results as the different pigeons were only exposed to either one or the other cage.

\section{RESULTS}

\section{Extinction}

Figure 1 shows the average pecks per session for the extinction (Ext) and control (Cnt) group of this experiment. Both groups showed typical and similar sensitization courses involving a response increment of about 3000 pecks $/ 20 \mathrm{~min}$, which did not differ significantly at asymptote (average over the three last sessions of the training phase: $P>0.05$ ). During the extinction phase the relatively weak CR of about 200 pecks/20 min which the Ext group initially showed in response to the CS cages diminished in the expected progressive way to an almost zero response level. Nevertheless, when tested for retention of the Apo sensitization in the same distinctive cages, their mean pecking scores over the three test sessions did not differ significantly from those of the Cnt group $(P>$ $0.05)$. This similarity between the Ext and Cnt groups was not due to a rapid reconditioning of the Exp group during the first test session, as no significant

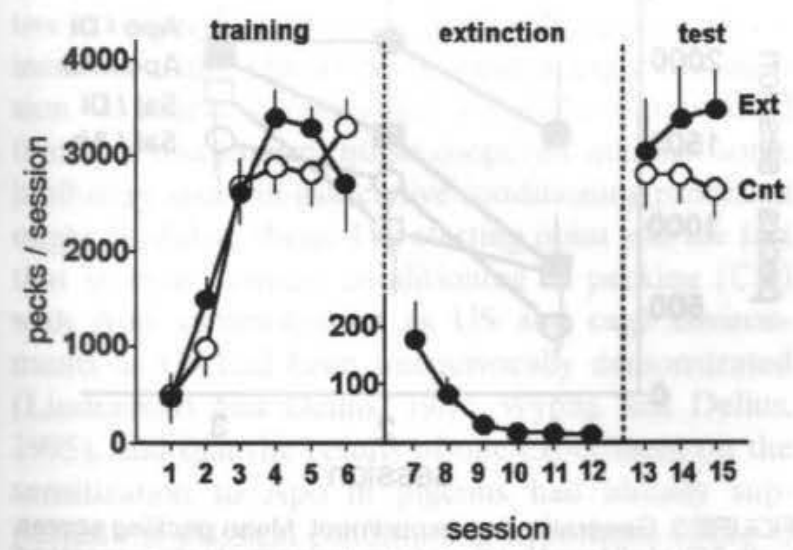

FIGURE 1. Extinction experiment. Mean pecking scores [with standard error (SE)] during the training, extinction and test phases of the extinction group (Ext) and control group (Cnt). Note the expanded pecks per session scale used for the extinction phase. difference was found between the groups' responses during the first $5 \mathrm{~min}$ of these sessions. In addition, neither group showed any significant change in pecking when the means of the last three training sessions were compared with the means of three test sessions $(P \mathrm{~s}>0.05)$. An intervening $\mathrm{CR}$ extinction thus seemed to have no detrimental effect on the sensitization increment induced during a preceding training with Apo.

\section{Conditioning}

The left half of Figure 2 shows the average pecks per session in the Di cage for all of the groups that were exposed to that cage during the training phase of this experiment. During the first four sessions of training the Apo-Di group showed the usual sensitization increase in pecking response. As expected, the Sal-Di and Di-Apo groups showed virtually no pecking in the Di cage during training. The animals of the latter group (as well as the Apo-Ho group) exhibited pecking bouts in their Ho cages after the Apo injections, but these responses were not recorded. However, as noted earlier, it is known that a full sensitization develops in pigeons which experience the Apo treatment only in their Ho cages (Wynne and Delius, 1995).

For the three sessions of the test phase, all the pigeons were injected with Apo and placed in the Di
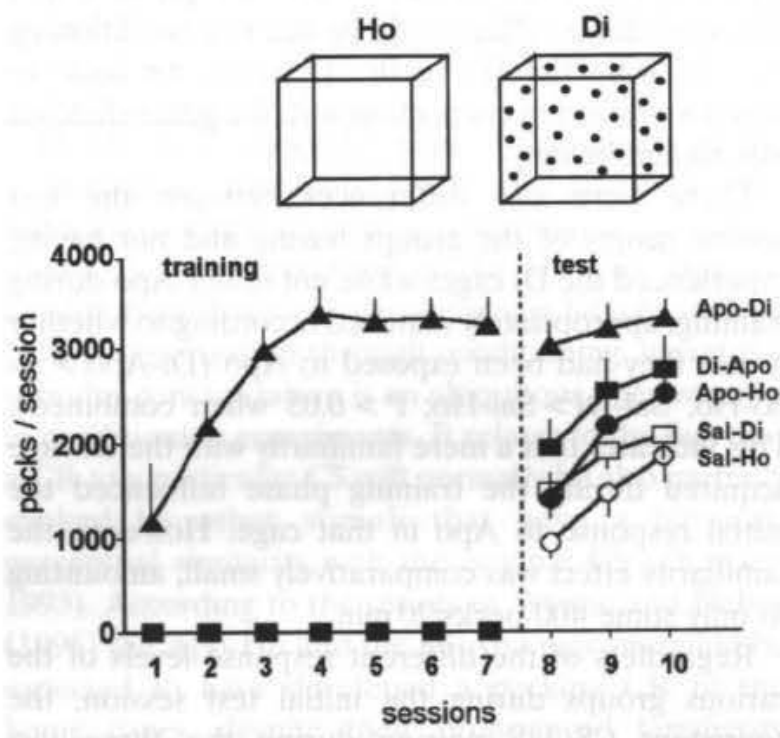

FIGURE 2. Conditioning experiment. Mean pecking scores (with SE) exhibited in the distinctive (Di) cages. During the training sessions (left), only groups Apo-Di, Di-Apo and SalDi were exposed to the Di cages. During the three test ses. sions (right), all five groups (including $\mathrm{Apo}-\mathrm{Ho}$ and $\mathrm{Sal}-\mathrm{Ho}$ ) were treated with Apo and exposed to the Di cages. Sketches of the home $(\mathrm{Ho})$ and $\mathrm{Di}$ cages used in this experiment are shown above. 
cage. During the critical first test session the various groups yielded different mean responses depending on how they had been previously trained. The ApoDi (paired CS-US) group, with the same treatment in training and test, yielded the strongest response, a straightforward continuation of the sensitized response that this group had reached by the end of the training phase. The Di-Apo group (unpaired CS-US) displayed a lower pecking response than the Apo-Di group $(P<0.05)$. The difference indicates that at least some of the sensitization exhibited by the ApoDi pigeons depended on the pairing between the CS and US during the training phase. This result is consistent with the conditioning hypothesis. However, the Di-Apo (unpaired US-CS) group showed a stronger response than the Sal-Di (CS-no-Us) group $(P>0.05)$, although not significantly so, and the Apo-Ho (US only) group exhibited a significantly stronger response than the control Sal-Ho group $(P<0.05)$. These differences, which are significant when appropriately combined (Di-Apo $>$ Sal-Di and Apo-Ho $>$ Sal-Ho; $P<0.05$ ) suggest that the presentation of the US alone, dissociated from the intended CS, was by itself capable of producing some sensitization. The Di-Apo > Apo-Ho group difference, incidentally, was not significant $(P>0.05)$. It would thus seem that a pharmacological process is implicated in the environment-dissociated sensitization increment exhibited by the Apo-Ho and Di-Apo trained groups. However, it is also still possible that this sensitization effect could be due to a conditioning transfer from the Ho to the Di cages, an issue to which we return in connection with the generalization experiment below.

There were also differences between the test session scores of the groups having and not having experienced the Di cages while not under Apo during training, appropriately matched according to whether or not they had been exposed to Apo (Di-Apo > Apo-Ho, Sal-Di $>$ Sal-Ho; $P>0.05$ when combined). This indicates that a mere familiarity with the Di cage acquired during the training phase influenced the initial response to Apo in that cage. However, the familiarity effect was comparatively small, amounting to only some 400 pecks $/ 20 \mathrm{~min}$.

Regardless of the different response levels of the various groups during the initial test session, the consistent CS-US pairing during the three test sessions yielded significant response increments ( $P \mathrm{~s}$ $<0.05$ ) from the first to the third sessions in all but the Apo-Di group which, in any case, was expected only to maintain the asymptotic responding already reached during training. Neither the familiarity which the Di-Apo and Sal-Di groups had with the Di cage, nor the novelty of this cage for the Sal-Ho and ApoHo groups, prevented the development of sensitization towards Apo in the testing environment.

\section{Generalization}

As all training for this experiment took place in the Ho cages, where pecking was routinely not recorded, Figure 3 displays only the results of test phases when the pigeons were treated with Apo in the test cages. Note that, as anticipated, the $\mathrm{Apo} / \mathrm{Di}$ and the $\mathrm{Sal} / \mathrm{Di}$ groups of the present experiment closely replicated the test results of the equivalent Apo-Ho and Sal-Ho groups of the conditioning experiment (Figure 2). Concerning the critical first session of the present experiment there was no significant difference between the $\mathrm{Sal} / \mathrm{Di}$ and the $\mathrm{Sal} / \mathrm{Ab}$ groups $(P>0.05)$. This result indicates that the $\mathrm{Di}$ and $\mathrm{Ab}$ test cages per se had no differential effect on the Apoelicited pecking. As could be anticipated, these Saltrained groups showed the lowest pecking responses during tests, irrespective of the cages used.

The Apo/Ab group exhibited a mean pecking response during the first test session which did not differ significantly from that of the control $\mathrm{Sal} / \mathrm{Ab}$ group $(P>0.05)$ but the Apo/Di group exhibited a stronger response, which did differ significantly from

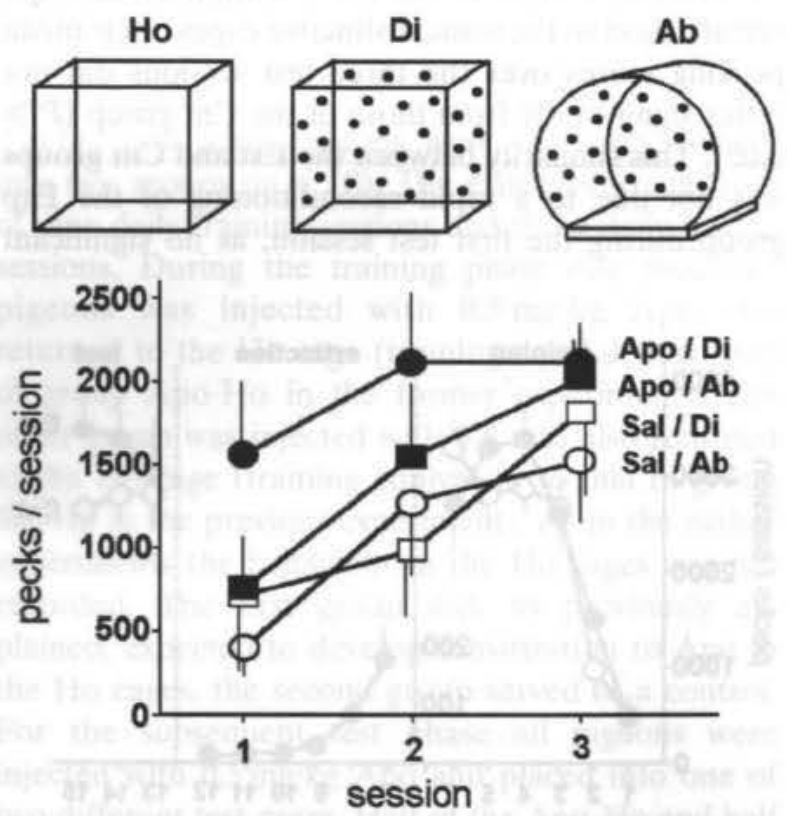

FIGURE 3. Generalization experiment. Mean pecking scores (with SE) of all four pigeon groups during the testing sessions after Apo injections in either the distinctive (Di) or the aber$\operatorname{rant}(\mathrm{Ab})$ cage. Sketches show the home $(\mathrm{Ho})$ cages in which the pigeons were injected either with Apo or Sal during the training phase, and the distinctive $\mathrm{Di}$ and $\mathrm{Ab}$ cages where they were all injected with Apo during testing. 
the control Sal/Di group $(P<0.05)$. This agreed with the expectation that during testing, the pecking $\mathrm{CR}$ to the Ho cages acquired during training would be less likely to transfer through stimulus generalization to the dissimilar $\mathrm{Ab}$ than to the similar $\mathrm{Di}$ cage. The alternative explanation that the relevant response difference arose because a non-associative familiarity with the training Ho cage had generalized more to the $\mathrm{Di}$ than to the Ab cage could be excluded. If this had been so, an analogous transfer of familiarity should have facilitated the first test session responding of the Sal/Di group more than that of the $\mathrm{Sal} / \mathrm{Ab}$ group. However, the $\mathrm{Sal} / \mathrm{Di}>\mathrm{Sal} / \mathrm{Ab}$ difference was far from significant $(P>0.05)$.

All four pigeon groups, incidentally, exhibited a significant increase in responding from the first to the third test session (Figure 3, $P \mathrm{~s}<0.05$ ). This no doubt reflected the sensitization that could be expected to develop due to the consistent drug-cage pairing that all the groups experienced during the test phase (compare the analogous results of the conditioning experiment).

\section{DISCUSSION}

We begin with the conclusions that can be drawn from the combined results of the conditioning and generalization experiments. Their purpose was to investigate whether the response increment (sensitization), that occurs when pigeons are repeatedly injected with a given dose of Apo in a given environment, might be due to the development of a pecking $\mathrm{CR}$ to the environmental CS, which progressively adds to the pecking UR elicited by the drug US. Or else, whether the sensitization might be due to a pharmacological process not involving associative excitatory conditioning, but involving some direct increase in the efficiency of dopaminergic transmission. Of course, the possibility should be kept in mind that both mechanisms might cooperate and that some inhibitory and non-associative conditioning processes might modulate them. The starting point was the fact that straight classical conditioning of pecking (CR) with Apo administration as US and cage environments as CS had been unequivocally demonstrated (Lindenblatt and Delius, 1987; Wynne and Delius, 1995), and that the results of one experiment on the sensitization to Apo in pigeons had already supported the classical conditioning hypothesis (Burg et al., 1989) but that the results of two other experiments (Wynne and Delius, 1995; present extinction experiment) had somewhat conflicted with this hypothesis.
The conditioning experiment examined the conditioning vs pharmacological accounts for sensitization using a design different from that employed by Burg et al. (1989). According to the exclusively pharmacological hypothesis, all of the groups of this experiment exposed to the drug US, regardless of whether it was paired with the CS or not (Apo-Di, Di-Apo and Apo-Ho groups), should have developed an approximately similar sensitization to Apo. Alternatively, according to the hypothesis based on conditioning, sensitization should only have occurred in the group trained with paired CS-US exposures, that is the Apo-Di group. Indeed, the pigeons of this latter group evinced more pecking upon testing than did those belonging to the Di-Apo and Apo-Ho groups. However, the two last groups still evinced a stronger response than the control Sal-Di and Sal-Ho groups. This latter result seemed to make it necessary to invoke a context-independent sensitization component, due perhaps to a pharmacological process. The full sensitization observed in the Apo-Di group thus seemed likely to be due to both a conditioned and a pharmacological component. The possibility that both these mechanisms would contribute to the Apo sensitization of pigeon pecking agreed with the conclusion reached by Mattingly and Gotsick (1989; see however Mattingly et al., 1997) concerning the Apo sensitization of the rat locomotor response after a partially similar experiment. It would also be in line with the widespread opinion that such a mixed process underlies the sensitization to cocaine and amphetamine in rodents (e.g. Burechailo and MartinIverson, 1996).

However, a reconsideration of the results of the conditioning experiment led to the alternative hypothesis that the supposedly pharmacological component of sensitization could still be due to conditioning. Even though seemingly largely ignored in the sensitization-through-conditioning literature, stimulus generalization is an ubiquitous phenomenon in conditioning experiments. It relates to the fact that a CR to a particular CS will normally be also partially evoked by other stimuli that have a physical/ perceptual similarity with the original CS (Domjan, 1993). According to the results of Wynne and Delius (1995) the Apo-Ho and the Di-Apo pigeons could be expected to have developed a pecking CR to the home cages, despite their pronounced familiarity with these cages. The distinctive and home cages were partially similar, as they had the same cubic shape and identical grid floors, fronts and ceilings. The pecking CR that the Apo-Ho and Di-Apo groups would have developed with respect to the home cages could have partly generalized to the distinctive cage 
when these groups were tested under the Apo-Di conditions. Accordingly, the increased test response of both groups could have been due to classical conditioning occurring during training followed by stimulus generalization occurring during testing, rather than to a pharmacological process.

To test this alternative we used an additional test cage which differed strongly from the home cages in the generalization experiment. This new aberrant cage was designed to minimize the incidence of generalization. As before, the home cages were used as training environments. The Sal-trained groups obviously displayed the lowest pecking response during the test, irrespective of the cage they were tested in. The generalization favouring the Apo/Di group of the present experiment replicated the sizeable and significant test pecking increment shown by the equivalent Apo-Ho-trained, Apo-Di-tested group of the former experiment. In contrast and in agreement with the expectation of minimal generalization, the $\mathrm{Apo} / \mathrm{Ab}$ group showed an insignificant pecking increment during the first testing session. Thus, the combined outcomes of the conditioning and generalization experiments fully support the provisional conclusion reached by Burg et al. (1989) that the sensitization of the pigeons' pecking response to Apo arises mainly through a classical conditioning mechanism. This conditioning mechanism, however, is unavoidably coupled to a potential stimulus generalization.

The extinction experiment attempted to assess the validity of the sensitization-through-conditioning hypothesis in a different way than to the conditioning and generalization experiments. It was based on the fact that any CR to a CS which had arisen through repeated CS-US pairings is expected to extinguish progressively when a series of CS-no-US presentations are instituted (Domjan, 1993). Accordingly, if the sensitization to Apo is due to conditioning, the response increment associated with it should wane when pigeons are repeatedly exposed without Apo to the same cage environment in which they were previously sensitized. That certainly did not happen, even though an extinction of the CR to the CS-noUS presentations was observed. The dissociation between the $\mathrm{CR}$ extinction and the retention of the sensitization increment is, at first sight, in contradiction with the conditioning hypothesis, and this result could thus be considered to support the pharmacological hypothesis. Note however, that the magnitude of the CR extinguished was much smaller than the sensitization increment obtained in the same experiment and that therefore the extinction procedure might not have had a detectable effect. But then the relatively small CR itself seems to be in contradiction with the hypothesis that the sizeable Apo sensitization increment is mainly due to the development of a CR to the environment in which the Apo effect was repeatedly experienced. We return to this problem later.

Meanwhile, it must be noted that this is not the first time that exposures to the CS-no-US condition were found to be ineffective in interfering with the sensitization to Apo in pigeons. Latent inhibition refers to the fact that repeated exposures to the CSno-US condition before the paired CS-US training usually inhibit the development of a CR to that CS. In two different experiments Wynne and Delius (1995) failed to find such an inhibitory effect on Apo sensitization. Repeated pre-exposure to a distinctive cage, or even to the home cage with Sal injection, did not interfere with the subsequent sensitization to Apo in the same cages. It thus seems reasonable to conclude that non-associative inhibitory conditioning plays no particular role in the Apo sensitization of pigeons. This, as far as a comparison is meaningful across different experimental designs, agrees with what Stewart and Vezina (1991) found concerning extinction using intact rats, amphetamine and locomotion, but disagrees with what Anagnostaras and Robinson (1996) found concerning latent inhibition using substantia nigra lesioned rats, amphetamine and turning.

From collateral results obtained in the conditioning experiment it appeared instead that an excitatory, non-associative conditioning (confusingly also called sensitization; Domjan, 1993) could play a modulatory role in pigeons. A preceding repeated exposure to the test cage (CS-no-US pre-treatment) appeared to have a minor facilitating role on the onset of the sensitization to Apo in pigeons, although of course it could not generate this by itself. This finding agrees, as far as comparison is again permissible, with Stewart and Vezina's finding (1991) that test environment familiarization enabled amphetamine sensitization in rats, but contrasts with Anagnostaras and Robinson's finding (1996) that a similar familiarization depressed the same responses. It may not be amiss, however, to remark that the distinction between non-associative and associative excitatory (and also inhibitory) conditioning may be less profound from the neurophysiological point of view than it has traditionally been considered from the behavioural point of view (Kandel et al., 1995).

The fact that latent inhibition and extinction do not impair the response increment that we suppose to be mainly due to a Pavlovian conditioning process, must be considered as somewhat unusual. However Apo, 
like amphetamine, cocaine and other psychoactive drugs, is also a somewhat unusual US. These substances can be expected to generate a drugdependent state that could act as an interoceptive CS component (Pert et al., 1990). Systemically injected Apo is known, or at least suspected, to excite or inhibit several different dopaminoceptive brain structures in birds, among them those responsible for the pecking UR (Lindenblatt and Delius, 1988; Wynne and Delius, 1996), but also structures that are part of the visual system such as the retina (Rohrer and Stell, 1995; cf. Brown and Makman, 1973: mammals). That is, Apo, as well as cocaine and amphetamine, are likely to modulate the perception of visual stimuli. Indeed, there is pharmacoethological evidence that a normally familiar environment acquires a markedly novel connotation for chickens when they are exposed to it under the influence of Apo (de Lanerolle and Millam, 1980). This implies that Apo may indeed have CS as well as US properties. Whenever a particular cage is used it may be necessary to distinguish between a $\mathrm{CS}_{\text {sal }}$ (cage perception under Sal influence) and a $\mathrm{CS}_{\text {apo }}$ (cage perception under Apo influence), that is, two notfully-identical versions of the same cage context. This idea applied to the results of the present experiment suggests that the $\mathrm{CR}_{\text {apo }}$ developed to the $\mathrm{CS}_{\text {apo }}$ during training can only partially be expected to extinguish during the repeated $\mathrm{CS}_{\text {sal }}$ presentations, i.e. only that fraction of the $\mathrm{CR}_{\text {apo }}$ which would generalize to the $\mathrm{CS}_{\text {sal }}$ (empirically about 200 pecks/ session). Such an extinction treatment would not appreciably weaken the complete $\mathrm{CR}_{\text {apo }}$ (empirically about 3000 pecks/session) to the $\mathrm{CS}_{\text {apo }}$ that was effective again during testing. Virtually the same sensitized response to Apo would thus be expected to re-emerge during the test phase in both the extinguished and the non-extinguished group. This explanation for the ineffectiveness of an extinction treatment is similar to that proposed by Wynne and Delius (1995) for the ineffectiveness of latent inhibition. The inhibitory conditioning that might have developed to the $\mathrm{CS}_{\mathrm{sal}}$ in their experiments would have only very partially generalized to the $\mathrm{CS}_{\text {apo. }}$.

This mechanism can also be used to explain why conditioned sensitization develops when pigeons repeatedly experience the effect of Apo in the home cages (Apo-Ho groups), even though this condition involves both CS-US and CS-no-US pairings as the subjects forcibly experience the same cage under both the Apo and the no-Apo state (Wynne and Delius, 1995). It clearly does not ensure the CS-US, no-CSno-US contingency generally considered essential for classical conditioning (Domjan, 1993). The point is that the proposed differential cage perceptions under Apo and no-Apo do, in fact, ensure the required conditioning contingency in a $\mathrm{CS}_{\mathrm{apo}}-\mathrm{US}$, no- $\mathrm{CS}_{\text {apo }}{ }^{-}$ no-US manner. The same process can also explain why the pecking $\mathrm{CR}_{\text {ho, }}$, which emerges from such conditioning, generalizes rather easily to other physically similar cages as, for example, from the home cage to the distinctive cage in the conditioning experiment. It is simply that similar cage environments can additionally come to share the Apo component, an extra similarity that further facilitates a CR generalization across such cages. Such a facilitated response transfer is likely to mimic major sensitization components, which prima facie would seem to be context-independent, and thus apparently due only to a pharmacological process. This mechanism also explains why, after CS-US training, the pecking CR constituting the sensitization increment is so much more intense than the pecking CR displayed in the CS-no-US situation (the 3000 vs 200 pecks/session highlighted earlier). The conditioned-only nature of the full Apo sensitization increment is hard to recognize because the CR behind it is due to a CS which is partially, yet inseparably, intertwined with the US. That may be one of the reasons why the sensitization-throughconditioning hypothesis has often been questioned. Mattingly and Gotsick (1989), for example, were sceptical whether this hypothesis applied to sensitization to Apo in rats, because the environment in which the sensitization treatment had taken place, when presented alone, elicited a locomotor response that was negligible when compared with the sensitization increment elicited by Apo in combination with the environment (cf. Anagnostaras and Robinson, 1996).

At this juncture it is proper to point out that we suspect that the CS which finally drives the effective sensitization to Apo in pigeons is a quite complex stimulus (cf. Pert et al., 1990: rodents). Apart from the interoceptive drug state and the visual cage environment, one must probably also consider the acoustic stimuli arising from the pigeons' own movements in different cages. Note, however, that we routinely controlled for the drug administration procedure in the sense that at the time of testing all groups had equal experience with it, using Sal injections where necessary. Nevertheless, this potential CS might have an interest of its own, as it seems possible that it could support a modulating inhibitory conditioning process in pigeons. This is suggested by the fact that the sensitization course at the start of training of the Apo-Di group, less habituated to the injection procedure, was possibly steeper than that of 
the more habituated Sal-Di and Sal-Ho groups during the test phase in the conditioning experiment. However, a better controlled experiment, where the two conditions would have to apply contemporaneously to two groups of pigeons, is needed to ascertain this.

Returning to the earlier argument, the assumption that two stimuli, the exteroceptive cage CS and the interoceptive drug US, interact in determining a finally effective CS may seem somewhat arbitrary. However, it has been recognized for some time that contextual stimuli can play an important role in classical conditioning (conditional context or occasion setting stimuli; Domjan, 1993). More recently, it has been accepted that even the US itself can provide such context cues. This idea postulates that, after conditioning, a given CS may elicit a CR in the presence of the US but the same CS may not do so in the absence of the US (Bouton, 1993). Killcross and Dickinson (1996) have, for example, demonstrated such an interaction between CS and US with a conventional tone-CS shock US-conditioning of rats, and have connected these results with a related effect which Killcross et al. (1994) observed with a latent inhibition procedure in amphetamine-treated rats. Whatever the precise mechanism, the CS and US interaction, varying perhaps on occasions from a mainly additive to a mainly multiplicative (configural: Pert et al., 1990) one, could perhaps signify the undoing of purported pharmacological components of drug sensitization. Nevertheless, it would be unwise at this stage to dismiss totally the intervention of a pharmacological process. With respect to pigeons at least, a similar effort as has been spent on developing the conditioning hypothesis has to be devoted to attempts to support the pharmacological hypothesis. Dopamine binding studies on pigeon brain tissue presently being carried out in our laboratory may help to clarify the situation. However, although the conditioning process has somehow to affect the properties of a neurotransmitter system, these need not necessarily be dopamine. Indeed, we rather suspect that mainly the glutamatergic system may be involved (Wickens, 1990; Cepeda and Levine, 1997). In any case, the Apo sensitization paradigm in pigeons seems to be a promising preparation for the study of the synapto-pharmacological bases of conditioning.

In this study we have defended the position that the sensitization to Apo found for the pecking response of pigeons is largely due to the intervention of a drug-induced, drug-dependent, context-contingent and stimulus generalization-prone excitatory classical conditioning process. We are uncertain whether this conclusion can be extended to other sensitization preparations. It cannot be automatically expected that precisely the same mechanisms need to underlie the sensitization to different drugs, in different species and different responses. It is necessary to remember that the avian brain differs considerably in structure and function from the mammalian one (Dubbledam, 1998). Clearly divergent results have in any case been found, even among mammals, concerning sensitization to the same drugs (Apo, amphetamine) and the same species (intact, nigra lesioned rats), but concerning different responses (locomotion or turning), with regard to whether a CR was observed in the CS-no-US condition (Carey, 1986; Mattingly and Gotsick, 1989), or whether a CS-no-US treatment facilitated, or suppressed, the development of sensitization (Stewart and Vezina, 1991; Anagnostaras and Robinson, 1996). Indeed, there are even somewhat contradictory results about whether the sensitization to Apo relating to the locomotor response of rats is in any way context-dependent (Mattingly and Gotsick, 1989; Mattingly et al. 1997). Nevertheless, the account that Anagnostaras and Robinson (1996) offer after a review of sensitization to psychostimulants in rodents, while lacking an explicit generalization component, has some similarities with our pigeon/apomorphine-based hypothesis. It seems possible that a more detailed consideration of the drug state and the stimulus generalization factors could help to streamline the interpretation of the evidence concerning sensitization in rodents. Perhaps the opinion that both conditioning and pharmacological mechanisms contribute in a multifactorial way to the sensitization to cocaine and amphetamine might have to yield to a sophisticated learning explanation. It might be worth considering that in nocturnal rodents, compared to diurnal birds, the intervention of hardto-control olfactory stimuli and their dopaminergically influenced central processing (Kandel et al., $1995)$ could even magnify the role of stimulus generalization and drug-state mechanisms. Whether the findings on pigeons and rodents can be integrated into a single sensitization theory is an issue that is beyond the purpose of this paper and one that, in our opinion, should wait until we know more about the sensitization in pigeons.

\section{Acknowledgements}

The research was supported by a grant from the Deutsche Forschungsgemeinshaft, Bonn to J.D. Delius. A.M. Godoy was a recipient of a Deutscher Akademischer Austauschdienst postgraduate fellowship. We thank M.J. Acerbo for much support, I. Krug for general assistance and Dr G.R. King 
(Duke University) for useful criticism of a draft. We are grateful to Prof. J. Emmerton (Purdue University) and to J. Grante (Exeter University) for improving the English. We are also grateful to an insistent anonymous referee for having forced us to improve the presentation and to refine our arguments.

\section{REFERENCES}

Andersen H, Braestrup C, Randrup A (1975). Apomorphineinduced biting in the tortoise in relation to dopaminergic mechanisms. Brain Behav Evol 11:365-372.

Acerbo MJ, Godoy AM, Delius JD (1997). Sensitisation of the apomorphine-induced pecking response in pigeons: learning or pharmacology? In: Göttinger neurobiology report. Elsner N, Wässle H (editor). Stuttgart: Thieme, p. 721.

Anagnostaras SG, Robinson TE (1996). Sensitization to the psychomotor stimulant effects of amphetamine modulation by associative learning. Behav Neurosci 110:1397-1414.

Basten-Kreft A (1977). Apomorphin-induziertes Verhalten bei Tauben (Apomorphine-induced behaviour in pigeons). Diplomthesis. Bochum: Ruhr-University.

Bedingfield JB, Calder LD, Karler R (1996). Comparative behavioral sensitization to sterotypy by direct and indirect dopamine agonists in CF-1 mice. Psychopharmacology 124:219-225.

Bell SM, Stewart RB, Thompson SC, Meisch RA (1997). Food deprivation increases cocaine-induced conditioned place preferences and locomotory activity in rats. Psychopharmacology 131:1-8.

Bouton ME (1993). Context, time, and memory retrieval in the interference paradigms of Pavlovian learning. Psychol Bull 114:80-99.

Brunelli M, Magni F, Moruzzi G, Musumeci D (1975). Apomorphine pecking in the pigeon. Arch Ital Biol 113:303-325.

Brown JH, Makman MH (1973). Influences of neuroleptic drugs and apomorphine on dopamine-sensitive adenylate cyclase of the retina. $J$ Neurochem 21:477-479.

Burechailo L, Martin-Iverson MT (1996). Behavioural sensitization to cocaine, but not cocaine-conditioned behaviour is asociated with increased dopamine occupation of ist receptors in the nucleus accumbens. Behav Neurosci 110:1388-1396.

Burg B, Haase C, Lindenblatt U, Delius JD (1989). Sensitization to and conditioning with apomorphine in pigeons. Biochem Pharmacol Behav 34:59-64.

Carey RJ (1986). Conditioned rotational behavior in rats with unilateral 6-hydroxydopamine lesions of the substantia nigra. Brain Res 356:379-382.

Cepeda C, Levine MS (1997). Dopamine and $N$-methyl-Daspartate receptor interactions in the neostriatum. Dev Neurosci 20:1-18.

Cheng HC, Bhatnager RK, Long JP (1975). Dopaminergic nature of amphetamine-induced pecking of pigeons. Eur $J$ Pharmacol 33:319-324.

Crombagh HS, Badiani A, Robinson TE (1996). Signalled versus unsignalled intravenous amphetamine: large differences in the acute psychomotor response and sensitization. Brain Res 722:227-231.

de Lanerolle NC, Millam JR (1980). Dopamine, chick behavior and states of attention. J Comp Physiol Psychol 94:346-352.

Delius JD (1985). The pecking of the pigeon: free for all. In: Behavior analysis and contemporary psychology. Lowe CF, Richelle M, Blackman DE, Bradshaw CM (editors). New York: Erlbaum, pp. 53-81.
Deviche P (1984). Administration of small doses of apomorphine attenuates feeding in non-deprived pigeons. Physiol Behav 33:581-585.

Domjan M (1993). The principles of learning and behavior (2nd ed.). Pacific Grove: Brooks/Cole.

Dubbledam JL (1998). Birds. In: The central nervous system of vertebrates. Nieuwenhuys R, Ten Donkelaar HJ, Nicholson C (editors). Berlin: Springer, pp. 1526-1636.

Fekete M, Kurti AM, Priubusz J (1970). On the dopaminergic nature of the gnawing compulsion induced by apomorphine in mice. $J$ Pharm Pharmacol 22:377-379.

Glagow M, Ewert J-P (1997). Dopaminergic modulation of visual responses in toads. J Comp Physiol A 180:1-9.

Julien RM (1995). A primer of drug action: a concise, nontechnical guide to the actions, uses, and side effects of psychoactive drugs. San Francisco, CA: Freeman.

Kalant H (1989). Drug tolerance and sensitization: a pharmacological overview. In: Psychoactive drugs, tolerance and sensitization. Goudie AJ, Emmet-Oglesby MW (editors). Clifton: Humana Press, pp. 547-577.

Kandel ER, Schwartz JH, Jessel TM (editors) (1995). Essentials of neural science and behavior. New York: Appleton and Lange.

Killcross S, Dickinson A (1996). Contextual control of latent inhibition by the reinforcer. Q J Exp Psychol, 49B:45-59.

Killcross S, Dickinson A, Robbins TW (1994). Amphetamineinduced disruptions of latent inhibition are reinforcer mediated. Psychopharmcology 115:185-195.

Kruk ZL, Pycock CJ (1991). Neurotransmitters and drugs (3rd ed.). London: Chapman and Hall.

Kuczenski R, Segal DS, Todd PK (1997). Behavioural sensitization and extracellular dopamine responses to amphetamine after various treatments. Psychopharmacology 134:221-219.

Kuribara H, Uchihashi Y (1993). Dopamine antagonists can inhibit methamphetamine sensitization but not cocaine sensitization when assesed by ambulatory activity in mice. J Pharm Pharmacol 45:1042-1045.

Lienau AK, Kuschinsky K (1997). Sensitization phenomena after repeated administration of cocaine and non-associative mechanisms and the role of dopamine in the striatum. Naunyn Schmiedebergs Arch Pharmacol 355:531-537.

Lindenblatt U, Delius JD (1987). Apomorphine-induced pecking in pigeons classically conditioned to environmental cues. Psychopharmcology 93:223-225.

Lindenblatt U, Delius JD (1988). Nucleus basalis prosencephali, a substrate of apomorphine-induced pecking in pigeons. Brain Res 453:1-8.

Ljungberg T, Ungerstedt U (1977). Apomorphine-induced locomotion and gnawing. Eur J Pharmacol 46:147-151.

Machlis L (1980). Apomorphine effects on the timing and sequencing of pecking behavior in chicks. Pharmacol Biochem Behav 13:331-336.

Mattingly BA, Gotsick JE (1989). Conditioning and experiential factors affecting the development of sensitization to apomorphine. Behav Neurosci 103:1311-1317.

Mattingly BA, Koch C, Osborne FH, Gotsick JE (1997). Stimulus and response factors affecting the development of behavioral sensitization to apomorphine. Psychopharmacology 130:109-116.

Pert A, Post R, Weiss SRB (1990). Conditioning as a critical determinant of sensitization induced by psychmotor stimulants. NIDA Res Monogr 97:208-241.

Rohrer B, Stell WS (1995). Localization of putative dopamine $\mathrm{D}_{2}$-like receptors in the chicken retina. Brain Res 695:110116.

Schwarting RKW, Huston JP (1996). The unilateral 6hydroxydopamine lesion model in behavioural brain re- 
search, analysis of functional deficits, recovery and treatments, Progr Neurobiology 50:275-331.

Siegel S, Castellan NJ (1988). Nonparametric statistics for the behavioral sciences. McGraw-Hill: New York.

Siemann M, Delius JD (1992a). Apomorphine-induced behaviour in pigeons (Columba livia). In: Rhythmogenesis in neurons and networks. Elsner N, Richter NR (editors). Stuttgart: Thieme, p. 600 .

Siemann M, Delius JD (1992b). Variability of forage pecking in pigeons. Ethology 92:29-50.

Stewart J, Badiani A (1993). Tolerance and sensitization to the behavioral effects of drugs. Behav Pharmacol 4:289-312.

Stewart J, Vezina P (1991). Extinction procedures abolish conditioned stimulus control but spare sensitized responding to apomorphine. Behav Pharmacol 2:65-71.

Szechtman H, Cleghorn JM, Brown GM, Kaplan RD, Franco SW, Rosenthal K (1987). Sensitization and tolerance to apomorphine in men: yawning, growth hormone, nausea, and hypothermia. Psychiatr Res 23:245-255.

White FJ, Joshi A, Koeltzow TE, Hu XT (1998). Dopamine receptor antagonist fails to prevent induction of cocaine sensitization. Neuropschopharmacology 18:26-40.

Wickens J (1990). Striatal dopamine in motor activation and reward-mediated learning: steps towards a unifying model. $J$ Neural Transm 80:9-31.

Winer BJ (1971). Statistical principles in experimental design (2nd ed.). New York: McGraw-Hill.

Wynne B, Delius JD (1995). Sensitization to apomorphine in pigeons: unaffected by latent inhibition but still due to classical conditioning. Psychopharmcology 119:414-420.

Wynne B, Delius JD (1996). Frontal forebrain lesions: effects on the foraging and apomorphine pecking of pigeons. Physiol Behav 59:757-762. 